\title{
Editorial
}

Karl-Heinz Paqué

\section{Wirtschaftsgeschichte: Neue Bedeutung für ein großes Fach}

DOI 10.1515/pwp-2014-0022

\section{Liebe Leserin, lieber Leser,}

die Weltfinanzkrise 2008 hatte viele Folgen. Eine davon ist der Wiederaufstieg eines traditionsreichen Faches, der Wirtschaftsgeschichte. Der Grund liegt auf der Hand: Die Weltwirtschaftskrise der dreißiger Jahre war mit einem Schlag nicht mehr nur ein vergangenes Ereignis von rein historischem Interesse, sondern ein Menetekel. Könnte so etwas vielleicht nochmals passieren? Und wenn ja, wie sollte die Politik reagieren? Was hatte sie damals falsch gemacht? Der Zufall wollte es, dass 2008 der Präsident der amerikanischen Zentralbank ausgerechnet Ben Bernanke war, also ein Professor der Volkswirtschaftslehre, der sich auch wissenschaftlich mit der „Great Depression“ der Zwischenkriegszeit intensiv beschäftigt hatte. An einem der großen Schalthebel geldpolitischer Macht saß also ein Kenner der Wirtschaftsgeschichte.

Heute wissen wir: Es kam nicht zu einer zweiten „Great Depression“ - auch wenn wir sechs Jahre nach dem spektakulären Zusammenbruch der Investment Bank Lehman Brothers noch immer an den gesamtwirtschaftlichen Folgen herum laborieren, vor allem in Europa. Dass es vergleichsweise glimpflich ausging, lag vielleicht auch an den Lehren der Weltwirtschaftskrise der dreißiger Jahre, also an der Arbeit und den Erkenntnissen der modernen Wirtschaftsgeschichte, deren Vertreter nicht nur Fakten sammeln und überprüfen, sondern auch die Welt analysieren und interpretieren, und zwar längst auch mit Hilfe ausgefeilter ökonometrischer Methoden. Es bleibt zu hoffen, dass auch die aktuelle Wissenschaftspolitik daraus Konsequenzen zieht: Wir brauchen in unseren Fakultäten Professuren für genau diese moderne Wirtschaftsgeschichte. Was an anglo-amerikanischen Universitäten selbstverständlich ist, muss auch im deutschsprachigen Raum zur Regel werden.

Karl-Heinz Paqué: Otto-von-Guericke-Universität Magdeburg, E-Mail: paque@ovgu.de

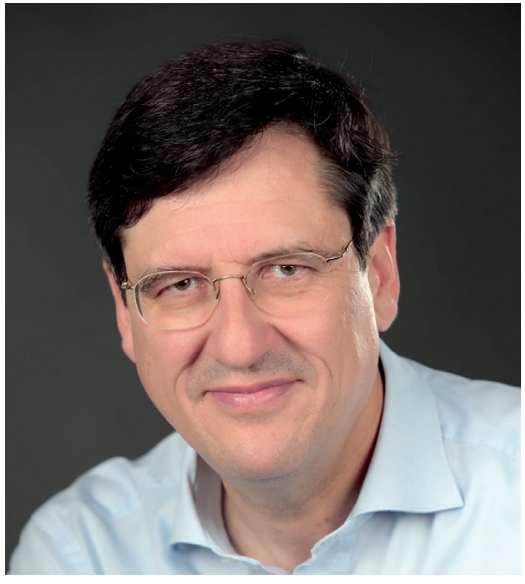

Jedenfalls werden die PWP in der Zukunft in der Rubrik „Wissenschaft im Überblick“ gelegentlich auch Beiträge aus der Wirtschaftsgeschichte präsentieren - vorausgesetzt natürlich, sie sind von wirtschaftspolitischer Bedeutung für die heutige Zeit. Einen Anfang machen in diesem Heft Mark Spoerer und Jochen Streb mit einem Beitrag über die Weimarer Republik in der Weltwirtschaftskrise. Diesem folgen Bernhard Boockmann, Claudia Buch und Monika Schnitzer mit dem abschließenden von drei Beiträgen zur evidenzbasierten Wirtschaftspolitik jenem Thema also, dem auch die diesjährige Jahrestagung des Vereins für Socialpolitik im September in Hamburg gewidmet war. Schließlich folgt eine Diskussion zu einem früheren Übersichtsartikel aus Heft 2 (2014), und zwar zum flächendeckenden Mindestlohn: Wolfram Richter kommentiert, die Autoren Andreas Knabe, Ronnie Schöb und Marcel Thum antworten. Es wird sicherlich nicht das letzte Mal sein, dass wir in der Rubrik „Wissenschaft im Überblick" eine Kontroverse veröffentlichen. Wir freuen uns nämlich darüber, wenn die Beiträge der PWP einen niveauvollen Meinungsaustausch provozieren. Genau so soll es sein - in einer lebendigen Gemeinschaft von Ökonomen und anderen Sozialwissenschaftlern.

Geoffrey Brennan, so lautet diesmal der Name des Interviewpartners in unserer Rubrik „Das Gespräch“. Ein anglo-amerikanischer, genauer: ein australischer Ökonom, und eine der großen Persönlichkeiten der Public 
Choice Theorie und Verfassungsökonomik, wie sie in der Tradition des 2013 verstorbenen Nobelpreisträgers der Wirtschaftswissenschaften James M. Buchanan seit den frühen sechziger Jahren entstanden ist und heute zum festen Bestandteil der finanz- und staatswissenschaftlichen Forschung und Lehre in unseren volkswirtschaftlichen Fachbereichen gehört. Das Interview führte Jan Schnellenbach, damals noch Mitarbeiter am Walter-Eucken-Institut Freiburg i. B. und inzwischen Professor für Volkswirtschaftslehre an der Brandenburgischen Technischen Universität Cottbus-Senftenberg.

Es folgen zwei referierte Beiträge aus der Forschung: Thiess Büttner, Wolfram Scheffler und Axel von Schwerin zur Hebesatzpolitik bei der Gewerbesteuer sowie Mark Andor, Manuel Frondel und Colin Vance zur Zahlungsbereitschaft für sogenannten grünen Strom. Den Abschluss des Heftes bildet die Thünen-Vorlesung der diesjährigen Jahrestagung des Vereins für Socialpolitik in Hamburg. Sie wurde von Walter Krämer gehalten. Ihr Titel: „Zur Ökonomie von Panik, Angst und Risiko“. Sie ist die erste publizierte Thünen-Vorlesung, seit die PWP in neuem Format erscheinen, aber sie wird nicht die letzte sein.
Auch für die Zukunft planen wir, die jährliche ThünenVorlesung in den PWP aufzunehmen, soweit sie einen wirtschaftspolitischen Bezug aufweist.

Vor Ihnen liegt nun das vierte Heft der PWP seit deren Neugestaltung. Der erste Jahrgang in neuem Gewande 2014 - ist damit komplett. Wir als Team der Herausgeber freuen uns über die vielen Reaktionen, die wir im Laufe dieses Jahres erhalten haben, ob zustimmend oder kritisch. Insgesamt können wir feststellen, dass die „neuen“ PWP anscheinend gut ankommen und das gewählte Format gefällt, äußerlich und inhaltlich. Wir hoffen, es bleibt so. Wir werden uns anstrengen. Dazu zählt auch, dass wir erkannte Schwächen beseitigen. Eine davon betrifft die bisherige Formulierung zur elektronischen Adresse für Einreichungen. Diese führte zu Missverständnissen. Daraus haben wir gelernt und formulieren jetzt wie folgt:

Manuskripte bitte unter http://mc.manuscriptcentral. com/pwp einreichen. Bei Rückfragen oder technischen Problemen wenden Sie sich bitte per E-Mail an die Geschäftsstelle: pwp@socialpolitik.org.

Karl-Heinz Paqué 